\title{
The effect of age and postweaning and adult handling habituation on activity and exploration in the rat
}

\author{
RICHARD W. THOMPSON and LOUIS G. LIPPMAN \\ Western Washington State College \\ Bellingham, Washington 98225
}

\begin{abstract}
Activity and exploratory behavior in rats was examined on 2 consecutive days of testing at 35 and again at 90 days of age using Greek cross mazes, proportional in size to the subject, having black, white, striped, and checkered arms. Half of the young animals received handling habituation prior to testing and half of each of these groups were handled prior to testing in adulthood. Young rats were more active than adults and handling, either postweaning or in adulthood, increased activity and exploratory behavior. Results also indicated that early handling potentiates the effects of later handling.
\end{abstract}

Although handling during infancy has been shown to increase later activity and exploratory behavior in rats (DeNelsky \& Denenberg, 1967a, 1967b; Schaefer, 1968; but see King, 1970), less is known about the effects of postweaning handling upon these behaviors. Meyers (1965) and Meyers, Leary, and Littman (1965) have found that postweaning handling will reduce the latency to enter a novel alley, suggesting an increase in exploratory tendency. Thompson and Lippman (1972), investigating activity and exploratory behavior in adult gerbils and rats using a Greek cross maze with black, white, striped, and checkered arms, found consistent results for rats for each of the three measures they employed. The three measures, compartment of first choice, frequency of compartment entrance, and a behavioral time sampling of each animal's location during each 180 -sec trial, indicated that rats preferred the black compartment, avoided the white, and showed a slight preference for the striped compartment over the white. Their experiments also indicated that habituation to handling in adulthood, repeated days of testing, and group housing resulted in higher activity scores and a trend toward more equal preference among compartments. In addition, Bronstein (1972) has shown that young rats habituated to handling are more active than adult rats habituated to handling, although he did not assess the effects of handling on activity per se.

The present experiment was designed to extend the investigation of the effects of handling habituation on activity and stimulus seeking to younger animals and also to assess whether postweaning handling habituation and repeated testing in a Greck cross maze will increase the effects of adult handling habituation on activity and exploratory behavior in a similar maze.

This research was supported by a grant from the Bureau for Faculty Research Western Washington State College. The authors wish to thank Steve Norsen, Deborah Wilson, and Larry Kronquist for their aid in data collection.

\section{METHOD}

\section{Subjects}

The subjects were 44 male albino rats 21 days of age obtained from Sprague-Dawley, Madison, Wisconsin. The animals were housed five to a cage and given ad-lib food and water throughout the experiment.

\section{Apparatus}

In an attempt to make the apparatus proportioned to the animal's size, young animals were tested in a Greek cross maze with arms $17 \mathrm{~cm}$ long, $12.5 \mathrm{~cm}$ wide, and $24 \mathrm{~cm}$ high. The floor of the maze was painted flat gray, the north and south arms were painted flat black and flat white, respectively. The east arm was painted with a 45-deg angle alternating $1.3-\mathrm{cm}$ wide black and white stripes, and the west arm was painted with $1.3-\mathrm{cm}$ square black and white checks. The maze was illuminated from above by two fluorescent lamps $210 \mathrm{~cm}$ above the floor. The adult animals were tested in a homologous but larger maze, more fully described in Thompson and Lippman (1972). The maze had arms $33 \mathrm{~cm}$ long, $25 \mathrm{~cm}$ wide, and $48 \mathrm{~cm}$ high. It was painted as described for the small maze except the stripes and checks were $2.5 \mathrm{~cm}$ wide.

\section{Procedure}

The experiment was conducted in two steps. In the first step, half the animals $(\mathrm{N}=22)$ received 10 days of handling habituation starting at 25 days of age and all animals were tested for 2 days in the maze at 35 days of age. In second step, the habituated and unhabituated groups were divided randomly, half of each group $(\mathrm{N}=11)$ received handling habituation for 10 days starting at 80 days of age and all animals were tested for 2 days in the maze at 90 days of age.

Handling habituation for both young and adult animals was identical to that described in Thompson and Lippman (1972). Briefly, handling consisted of removing the home cage from the cage rack, transferring each animal, one at a time, to an identical cage, and then returning the animals to the cage and the cage to the cage rack. This was repeated five times each day so that each animal was handled a total of 100 times. Each animal was tested individually and given a 3 -min trial on each of 2 consecutive days. The subject was placed in the center of the maze facing one of the compartments. The compartment which a subject faced was systematically rotated so that subjects were placed facing each compartment equally often. Three measures were taken: (a) which compartment the subject chose to enter first, (b) the frequency each subject entered cach compartment, and 
(c) which compartment the subject was in at the end of each $10 \mathrm{sec}$ of the $180-\mathrm{sec}$ trial.

\section{RESULTS}

The data gathered in the two parts of the experiment, that for the young and for the adult animals, were analyzed separately for each of the three measures employed: (a) first entry, (b) frequency of entrance, and (c) time sampling. A third set of analyses, one for each measure, provided comparisions between performance of young and adult animals. Initial analysis was made by analysis of variance. Subsequent analysis of significant interactions were by analysis of variance and/or Duncan's multiple range tests. Only effects significant at $\mathrm{p}<.05$ are reported.

\section{Young Animals}

Analysis of variance of the three measures used a between factor of handling or no handling and within factors of days and compartments.

First entry. The young animals revealed no compartment preference on this measure.

Frequency of entrance. The analysis indicated that the handled subjects were more active than the unhandled. The analysis also revealed a significant compartment effect and Compartment by Days interaction; the data for the interaction (combining over handling conditions) are presented in Figure 1. Although the black compartment was the most frequently entered compartment on both test days, the differential ordering of compartments seen on Day 1 (black, checks, stripes, and white), was absent on Day 2 .

Time sampling. Analysis of the time sampling data revealed a compartment effect and Handling by Compartment interaction, which is presented in Figure 2 (combining the 2 days of testing). Although this figure indicates that both handled and unhandled rats were

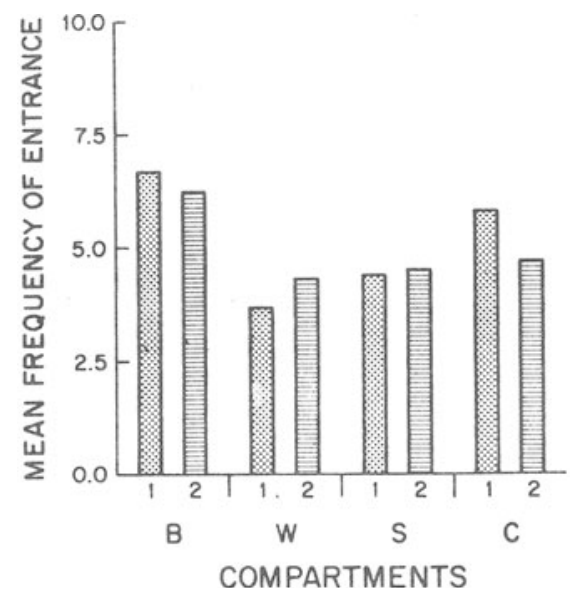

Figure 1. Mean frequency of compartment entrance for each compartment over the 2 days of testing, the Compartment by Days interaction.

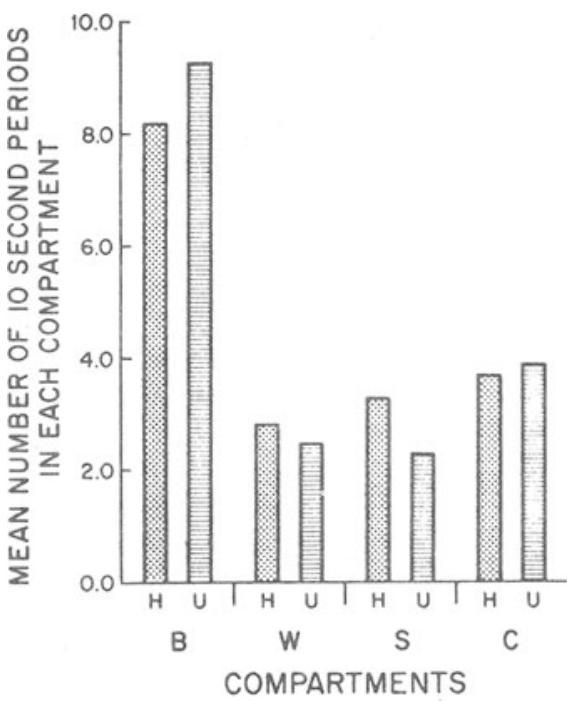

Figure 2. The mean number of time samples that handled and unhandled subjects were observed in each compartment, the Handling by Compartment interacton.

most likely to be found in the black compartment and less likely to be found in white, handling habituation tended to equate compartment preferences.

\section{Adult Animals}

Analysis of data gathered on the adult animals employed between factors of early handling and late handling and within factors of days and compartments.

First entry. This analysis indicated that the black compartment was entered first more often than white or checks.

Frequency. Analysis of these data revealed significant effects of early handling and compartments plus interactions of Late Handling by Days, Early Handling by Late Handling by Days and Early Handling by Days by Compartments.

The Early Handling by Late Handling by Days interaction was analyzed further via separate analyses of variance for subjects receiving early handing or no early handling, the factors in each analysis being late handling and days. For subjects receiving no early handling, adult handled animals had a higher frequency of compartment entrance than animals not handled in adulthood. For subjects receiving early handling there was a decrease in frequency of compartment entrance from Day 1 to Day 2 only for late handled subjects.

The Early Handling by Days by Compartment interaction (combining over late handling conditions) is presented in Figure 3. To clarify the source of this interaction, a separate analysis of variance was computed on subjects that received or did not receive postweaning handling, the factors in each analysis being Days and Compartments. Results of these analyses indicated that adult animals given postweaning handling habituation approached low intensity stimulation and avoided high 


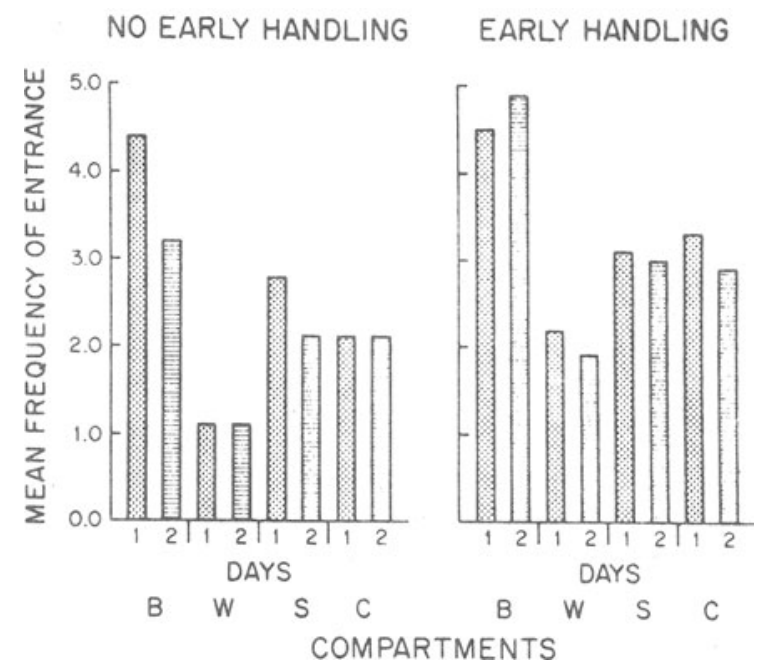

Figure 3. The mean frequency of entrance into each compartment over both days of testing for early handled and unhandled subjects, the Early Handling by Days by Compartment interaction.

intensity stimulation irrespective of complexity. Animals not given postweaning handling, however, also showed avoidance of high complexity, with black entered more frequently than checks or stripes, and black, checks, and stripes all entered more often than white. Comparison of each compartment between the 2 days of testing revealed only a decrease in compartment entrances for the black compartment.

Time sampling. Analysis of these data revealed only a significant compartments effect. Comparisons among the compartments indicated that adult animals show a consistent preference for black, and avoidance of the white compartment, with equal and intermediate preference for the checks and striped compartments.

\section{Young and Adult Animals}

For each measure, an analysis of variance was computed with factors of age, early handling, late handling, compartments, and days.

First entry. Only the Age by Compartment interaction was significant, indicating that only the adult animals showed a compartment preference, that for the black compartment.

Frequency. The significant main effect of age indicated that young animals were more active than adults, thus supporting and extending the generality of Bronstein's (1972) results based on open field activity. This analysis also yielded significant main effects of early handling, days, and compartments plus interactions of Age by Compartments, Days by Compartments, Age by Days by Compartments and Early Handling by Late Handling by Days.

The Early Handling by Late Handling by Days interaction, which is presented in Table 1 , can be explained by noting that this interaction was
Table 1

Mean Frequency of Compartment Entrance, Combining Over Age and Compartments, the Early Handling by Late Handling by Days Interaction

\begin{tabular}{llcc}
\hline & & Day 1 & Day 2 \\
\hline \multirow{2}{*}{ Early Handled } & Late Handled & 4.63 & 3.98 \\
& Late Unhandled & 4.07 & 4.42 \\
\multirow{2}{*}{ Early Unhandled } & Late Handled & 3.81 & 3.47 \\
& Late Unhandled & 3.67 & 3.21 \\
\hline
\end{tabular}

nonsignificant for data on young animals but was significant for adult animals even though the trends were similar in both sets of data. As is apparent in Table 2, the Age by Days by Compartment interaction indicates differential frequency of entrance into the various compartments across days for the two ages. Further analysis indicated that for young animals there was an ordering of frequency of compartment entrance on Day 1; black was entered most frequently, followed by checks and stripes, and white was avoided. Although there was still greater entrance into black than any other compartment on Day 2, the other compartments were entered equally often. The adult animals behaved uniformly on both days of testing, entering black most often, avoiding white, and entering the ckeckered and striped compartments equally often. Table 2 shows also that the young animals are clearly more active than the adults.

Time sampling. The analysis revealed a significant effect of compartments and interactions of Age by Compartments and Age by Late Handling by Compartments. Since subsequent analyses of the Age by Late Handling by Compartment interaction at each age revealed no significant main effects or interactions, the small difference among compartments as a function of age levels and handling conditions do not warrant interpretation. Differential compartment preferences at each age level have been specified above.

\section{DISCUSSION}

Results of the initial test series (young animals) showed that postweaning handling increases exploratory behavior and activity. Compartment preference, as indicated by time-sampling data, indicated that despite a clear preference for black and avoidance of white, handling tended to equate young animals' preferences among the striped and checkered compartments.

Table 2

Mean Frequency of Compartment Entrance, Combining Over Both Early and Late Handling Conditions, the Age by Days by Compartments Interaction

\begin{tabular}{|c|c|c|c|c|c|}
\hline & & \multicolumn{4}{|c|}{ Compartments } \\
\hline & & B & W & $S$ & $\mathrm{C}$ \\
\hline \multirow{2}{*}{ Young Rats } & Day 1 & 6.66 & 3.71 & 4.41 & 5.84 \\
\hline & Day 2 & 6.18 & 4.25 & 4.46 & 4.66 \\
\hline \multirow{2}{*}{ Adult Rats } & Day 1 & 4.41 & 1.66 & 2.96 & 2.71 \\
\hline & Day 2 & 4.05 & 1.46 & 2.57 & 2.52 \\
\hline
\end{tabular}


With the single exception of a reversal in preference among striped and checkered compartments for unhandled animals, these results are identical to those reported for adult animals by Thompson and Lippman (1972).

As with the young animals, handling in adult animals also increased activity, but did not affect compartment preference, as measured by time sampling, during the second test series. Thus, the two test series yielded similar results which, in turn, were consistent with prior results based on animals tested only in adulthood (Thompson \& Lippman). For all measures, the black compartment was preferred, white was avoided, and striped and checkered compartments represented weak intermediate preferences.

The potentiating or residual effects of early handling on adult behavior was detectable only on the activity measure. The group receiving both postweaning and adult handling showed a decrease in activity from Day 1 to Day 2; there were no changes for the other groups. Thompson and Lippman (1972) observed an increase in activity over the 2 days of testing which they attributed to a decrease in emotionality. If it is assumed that handling habituation decreases emotionality, then it would be expected that the group given both early and late handling would have been less emotional than any other group. It is suggested that the increased activity of animals handled both early and late would have brought them into greater commerce with the stimuli of the maze and provided opportunity for greater satiation to the maze elements, which yielded the decrease in exploratory (activity) tendencies over the 2 days of adult testing. Handling habituation at either postweaning or in adulthood will decrease emotionality and increase exploratory behavior. Providing habituation to handling at both times accelerates the decline in emotionality, increases exploration and eventual satiation to the stimuli in that context.

\section{REFERENCES}

Bronstein, P. M. Open-field behavior of the rat as a function of age: Cross-sectional and longitudinal investigations. Journal of Comparative and Physiological Psychology, 1972, 80, 335-341.

DeNelsky, G. Y., \& Denenberg, V. H. Infantile stimulation and adult exploratory behavior: Effects of handling upon tactual variation seeking. Journal of Comparative and Physiological Psychology, 1967a, 63, 309-312.

DeNelsky, G. Y., \& Denenberg, V. H. Infantile stimulation and adult exploratory behavior in the rat: Effects of handling upon visual variation seeking. Animal Behavior, 1967b, 15, 568-573.

King, D. L Effect of early experience and litter on some emotional variables in the rat. Journal of Comparative and Physiological Psy chology, 1970, 73, 436-441.

Meyers, W. J. Effects of different intensities of postweaning shock and handling on the albino rat. Journal of Genetic Psy chology, 1965, 106, 51-58.

Meyers, W. J., Leary, R. W., \& Littman, R. A. Free-chnoice exploration: Decreased responsivity to illumination from prior experience. Journal of Comparative and Physiological Psy chology, 1965, 60, 297-298.

Schaefer, Jr. T. Some methodological implications of research on "Early handling" in the rat. In G. Newton, and S. Levine (Eds.), Early experience and behavior, Springfield: Thomas, 1968.

Thompson, R. W, \& Lippman, L, G. Exploration and activity in the gerbil and rat, Journal of Comparative and Physiological Psychology, 1972, 80, 439-448.

(Received for publication December 23, 1974.) 